\title{
Tetrahedra on deformed spheres and integral group cohomology
}

\author{
Pavle V. M. Blagojević ${ }^{*}$ \\ Mathematički Institut \\ Knez Michailova 35/1 \\ 11001 Beograd, Serbia \\ pavleb@mi.sanu.ac.rs
}

\author{
Günter M. Ziegler** \\ Inst. Mathematics, MA 6-2 \\ TU Berlin \\ D-10623 Berlin, Germany \\ ziegler@math.tu-berlin.de
}

Submitted: Aug 28, 2008; Accepted: Jun 4, 2009; Published: Jun 10, 2009

Mathematics Subject Classification: 55S91, 55M20, 52C99

Dedicated to Anders Björner on the occasion of his 60th birthday

\begin{abstract}
We show that for every injective continuous map $f: S^{2} \rightarrow \mathbb{R}^{3}$ there are four distinct points in the image of $f$ such that the convex hull is a tetrahedron with the property that two opposite edges have the same length and the other four edges are also of equal length. This result represents a partial result for the topological Borsuk problem for $\mathbb{R}^{3}$. Our proof of the geometrical claim, via Fadell-Husseini index theory, provides an instance where arguments based on group cohomology with integer coefficients yield results that cannot be accessed using only field coefficients.
\end{abstract}

\section{Introduction}

The motivation for the study of the existence of particular types of tetrahedra on deformed 2-spheres is twofold. The topological Borsuk problem, as formulated by Soibelman in 1977 [6] ("estimate the minimal Borsuk partition number for the unit ball in $\mathbb{R}^{n}$ for general metrics!"), along with the square peg problem [5] first posed by Toeplitz 1911 ("does every Jordan curve contain the vertices of a square?") inspire the search for possible polytopes with nice metric properties whose vertices lie on the continuous images of spheres. Beyond their intrinsic interest, these problems can be used as testing grounds for tools from equivariant topology, e.g. for comparing the strength of Fadell-Husseini index theory with ring resp. field coefficients.

\footnotetext{
*Supported by the grant 144018 of the Serbian Ministry of Science and Technological development

** Supported by the German Research Foundation DFG
} 
The following theorem will be proved through the use of Fadell-Husseini index theory with coefficients in the ring $\mathbb{Z}$. It is also going to be demonstrated that Fadell-Husseini index theory with coefficients in field $\mathbb{F}_{2}$ has no power in this instance (Section 4.1).

Theorem 1.1. Let $f: S^{2} \rightarrow \mathbb{R}^{3}$ be an injective continuous map. Then its image contains vertices of a tetrahedron that has at least the symmetry of a square. That is, there are four distinct points $\xi_{1}, \xi_{2}, \xi_{3}$ and $\xi_{4}$ on $S^{2}$ such that

$$
d\left(f\left(\xi_{1}\right), f\left(\xi_{2}\right)\right)=d\left(f\left(\xi_{2}\right), f\left(\xi_{3}\right)\right)=d\left(f\left(\xi_{3}\right), f\left(\xi_{4}\right)\right)=d\left(f\left(\xi_{4}\right), f\left(\xi_{1}\right)\right)
$$

and

$$
d\left(f\left(\xi_{1}\right), f\left(\xi_{3}\right)\right)=d\left(f\left(\xi_{2}\right), f\left(\xi_{4}\right)\right) .
$$

Thus the tetrahedron may even be regular and thus have symmetry group $S_{4}$; it may also degenerate to a (planar) square.

Remark 1.2. The proof is not going to use any properties of $\mathbb{R}^{3}$ except that it is a metric space. Thus in the statement of the theorem, $\mathbb{R}^{3}$ can be replaced by any metric space $(M, d)$.

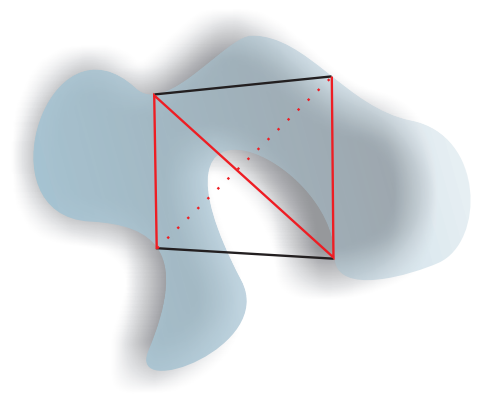

Figure 1: A $D_{8}$-invariant tetrahedron on a deformed 2-sphere

Let us try to relate this to the square peg problem and the topological Borsuk problem:

The square peg problem is settled for various classes of sufficiently piecewise-smooth Jordan curves, but open in general. Unfortunately, the methods used for the proof of Theorem 1.1] do not imply any conclusion when applied to the square peg problem (see Section 4.2). On the other hand, if the square peg problem could be solved for the continuous Jordan curves, then it would imply the result of Theorem 1.1.

The first open instance of the topological Borsuk problem considers the existence of a collection of four points with equal pairwise $d$-distances in a general metric space $\left(\mathbb{R}^{3}, d\right)$. The main result of the paper does not provide any new information concerning the topological Borsuk problem, as we work in the restricted parameter space $\left\{\left(x_{1}, x_{2}, x_{3}, x_{4}\right) \in\right.$ $\left(S^{1}\right)^{4} \mid x_{1} \neq x_{3}$ or $\left.x_{2} \neq x_{4}\right\}$ whose dimension is much smaller compared to the one used in the topological Borsuk problem $\left\{\left(x_{1}, x_{2}, x_{3}, x_{4}\right) \in\left(\mathbb{R}^{3}\right)^{4} \mid x_{1} \neq x_{3}\right.$ or $\left.x_{2} \neq x_{4}\right\}$. 


\section{Introducing the equivariant question}

Let $f: S^{2} \rightarrow \mathbb{R}^{3}$ be an injective continuous map. Denote by $D_{8}$ the symmetry group of a square, that is, the 8-element dihedral group $D_{8}=\left\langle\omega, j \mid \omega^{4}=j^{2}=1, \omega j=j \omega^{3}\right\rangle$.

\section{A few $D_{8}$-representations.}

The real vector spaces

$$
\begin{aligned}
& U_{4}=\left\{\left(x_{1}, x_{2}, x_{3}, x_{4}\right) \in \mathbb{R}^{4} \mid x_{1}+x_{2}+x_{3}+x_{4}=0\right\} \\
& U_{2}=\left\{\left(x_{1}, x_{2}\right) \in \mathbb{R}^{2} \mid x_{1}+x_{2}=0\right\}
\end{aligned}
$$

are real $D_{8}$-representations with actions given by

(a) for $\left(x_{1}, x_{2}, x_{3}, x_{4}\right) \in U_{4}$ :

$$
\omega \cdot\left(x_{1}, x_{2}, x_{3}, x_{4}\right)=\left(x_{2}, x_{3}, x_{4}, x_{1}\right), \quad j \cdot\left(x_{1}, x_{2}, x_{3}, x_{4}\right)=\left(x_{3}, x_{2}, x_{1}, x_{4}\right),
$$

(b) for $\left(x_{1}, x_{2}\right) \in U_{2}$ :

$$
\omega \cdot\left(x_{1}, x_{2}\right)=\left(x_{2}, x_{1}\right), \quad j \cdot\left(x_{1}, x_{2}\right)=\left(x_{2}, x_{1}\right),
$$

\section{The configuration space.}

Let $X=S^{2} \times S^{2} \times S^{2} \times S^{2}$ and let $Y$ be the subspace given by

$$
Y=\left\{(x, y, x, y) \mid x, y \in S^{2}\right\} \approx S^{2} \times S^{2} .
$$

The configuration space to be considered is the space

$$
\Omega:=X \backslash Y
$$

Let a $D_{8}$-action on $X$ be induced by

$$
\omega \cdot\left(\xi_{1}, \xi_{2}, \xi_{3}, \xi_{4}\right)=\left(\xi_{2}, \xi_{3}, \xi_{4}, \xi_{1}\right), \quad j \cdot\left(\xi_{1}, \xi_{2}, \xi_{3}, \xi_{4}\right)=\left(\xi_{4}, \xi_{3}, \xi_{2}, \xi_{1}\right),
$$

for $\left(\xi_{1}, \xi_{2}, \xi_{3}, \xi_{4}\right) \in X$.

\section{A test map.}

Let $\tau: \Omega \rightarrow U_{4} \times U_{2}$ be a map defined for $\left(\xi_{1}, \xi_{2}, \xi_{3}, \xi_{4}\right) \in X$ by

$$
\tau\left(\xi_{1}, \xi_{2}, \xi_{3}, \xi_{4}\right)=\left(d_{12}-\frac{\Delta}{4}, d_{23}-\frac{\Delta}{4}, d_{34}-\frac{\Delta}{4}, d_{41}-\frac{\Delta}{4}\right) \times\left(d_{13}-\frac{\Phi}{2}, d_{24}-\frac{\Phi}{2}\right)
$$

where $d_{i j}=d_{j i}:=d\left(f\left(\xi_{i}\right), f\left(\xi_{j}\right)\right)$ and

$$
\Delta=d_{12}+d_{23}+d_{34}+d_{14}, \quad \Phi=d_{13}+d_{24} .
$$


With the $D_{8}$-actions introduced above the test map $\tau$ is $D_{8}$-equivariant. Indeed,

$$
\begin{aligned}
\tau\left(\omega \cdot\left(\xi_{1}, \xi_{2}, \xi_{3}, \xi_{4}\right)\right) & =\tau\left(\xi_{2}, \xi_{3}, \xi_{4}, \xi_{1}\right) \\
& =\left(d_{23}-\frac{\Delta}{4}, d_{34}-\frac{\Delta}{4}, d_{41}-\frac{\Delta}{4}, d_{12}-\frac{\Delta}{4}\right) \times\left(d_{24}-\frac{\Phi}{2}, d_{13}-\frac{\Phi}{2}\right) \\
& =\omega \cdot\left(\left(d_{12}-\frac{\Delta}{4}, d_{23}-\frac{\Delta}{4}, d_{34}-\frac{\Delta}{4}, d_{41}-\frac{\Delta}{4}\right) \times\left(d_{13}-\frac{\Phi}{2}, d_{24}-\frac{\Phi}{2}\right)\right)
\end{aligned}
$$

and

$$
\begin{aligned}
\tau\left(j \cdot\left(\xi_{1}, \xi_{2}, \xi_{3}, \xi_{4}\right)\right) & =\tau\left(\xi_{4}, \xi_{3}, \xi_{2}, \xi_{1}\right) \\
& =\left(d_{43}-\frac{\Delta}{4}, d_{32}-\frac{\Delta}{4}, d_{21}-\frac{\Delta}{4}, d_{14}-\frac{\Delta}{4}\right) \times\left(d_{42}-\frac{\Phi}{2}, d_{31}-\frac{\Phi}{2}\right) \\
& =j \cdot\left(\left(d_{12}-\frac{\Delta}{4}, d_{23}-\frac{\Delta}{4}, d_{34}-\frac{\Delta}{4}, d_{41}-\frac{\Delta}{4}\right) \times\left(d_{13}-\frac{\Phi}{2}, d_{24}-\frac{\Phi}{2}\right)\right) .
\end{aligned}
$$

The following proposition connects our set-up with the tetrahedron problem.

Proposition 2.1. If there is no $D_{8}$ equivariant map

$$
\alpha: \Omega \rightarrow\left(U_{4} \times U_{2}\right) \backslash(\{\mathbf{0}\} \times\{\mathbf{0}\})
$$

then Theorem 1.1 follows.

Proof. If there is no $D_{8}$ equivariant map $\Omega \rightarrow\left(U_{4} \times U_{2}\right) \backslash(\{\mathbf{0}\} \times\{\mathbf{0}\})$, then for every continuous embedding $f: S^{2} \rightarrow \mathbb{R}^{3}$ there is a point $\xi=\left(\xi_{1}, \xi_{2}, \xi_{3}, \xi_{4}\right) \in \Omega=X \backslash Y$ such that

$$
\tau\left(\xi_{1}, \xi_{2}, \xi_{3}, \xi_{4}\right)=(\mathbf{0}, \mathbf{0}) \in U_{4} \times U_{2} .
$$

From (3) we conclude that

$$
d_{12}=d_{23}=d_{34}=d_{14}=\frac{\Delta}{4} \quad \text { and } \quad d_{13}=d_{24}=\frac{\Phi}{2} .
$$

It only remains to prove that all four points are different. Since $\left(\xi_{1}, \xi_{2}, \xi_{3}, \xi_{4}\right) \notin Y$ we have $\xi_{1} \neq \xi_{3}$ or $\xi_{2} \neq \xi_{4}$. By symmetry we may assume that $\xi_{1} \neq \xi_{3}$. The map $f$ is injective, therefore $f\left(\xi_{1}\right) \neq f\left(\xi_{3}\right)$ and consequently $d_{13} \neq 0$. Now

$$
d_{13} \neq 0 \Rightarrow d_{24} \neq 0 \Rightarrow f\left(\xi_{1}\right) \neq f\left(\xi_{3}\right), f\left(\xi_{2}\right) \neq f\left(\xi_{4}\right) \Rightarrow \xi_{1} \neq \xi_{3}, \xi_{2} \neq \xi_{4} .
$$

Let us assume, without loss of generality, that $\xi_{1}=\xi_{2}$. Then $d_{12}=d_{23}=d_{34}=d_{14}=0$, which implies that $d_{13} \leq d_{12}+d_{23}=0$. This yield a contradiction to $d_{13} \neq 0$. Thus $\xi_{1} \neq \xi_{2}$.

The unit sphere of the representation $U_{4} \times U_{2}$ will be denoted by $S\left(U_{4} \times U_{2}\right)$. Notice that there is a $D_{8}$-equivariant deformation of $\left(U_{4} \times U_{2}\right) \backslash(\{\mathbf{0}\} \times\{\mathbf{0}\})$ onto the sphere $S\left(U_{4} \times U_{2}\right)$. Thus, there are $D_{8}$-equivariant maps $\left(U_{4} \times U_{2}\right) \backslash(\{\mathbf{0}\} \times\{\mathbf{0}\}) \rightarrow S\left(U_{4} \times U_{2}\right)$ and $S\left(U_{4} \times U_{2}\right) \rightarrow\left(U_{4} \times U_{2}\right) \backslash(\{\mathbf{0}\} \times\{\mathbf{0}\})$. Hence by Proposition 2.1. Theorem 1.1] is a consequence of the following topological result.

Theorem 2.2. There is no $D_{8}$-equivariant map $\Omega \rightarrow S\left(U_{4} \times U_{2}\right)$.

Indeed, we will prove a stronger result: There is no $\mathbb{Z}_{4}$-equivariant map $\Omega \rightarrow S\left(U_{4} \times U_{2}\right)$. 


\section{Proof of Theorem 2.2}

The proof is going to be conducted through a comparison of the Serre spectral sequences with $\mathbb{Z}$-coefficients of the Borel constructions associated with the spaces $\Omega$ and $S\left(U_{4} \times U_{2}\right)$ and the subgroup $\mathbb{Z}_{4}=\langle\omega\rangle$ of $D_{8}$. In other words, we determine the $\mathbb{Z}_{4}$ Fadell-Husseini index of these spaces living in $H^{*}\left(\mathbb{Z}_{4} ; \mathbb{Z}\right)=\mathbb{Z}[U] / 4 U$, $\operatorname{deg} U=2$.

The Fadell-Husseini index of a $G$-space $X$, Index $\operatorname{lex}_{G, \mathbb{Z}} X$, is the kernel of the map

$$
\pi_{X}^{*}: H^{*}(\mathrm{~B} G, \mathbb{Z}) \rightarrow H^{*}\left(X \times_{G} \mathrm{E} G, \mathbb{Z}\right)
$$

induced by the projection $\pi_{X}: X \times_{G} \mathrm{E} G \rightarrow \mathrm{B} G$. Consider a $G$-equivariant map $f: X \rightarrow Y$ between two $G$-spaces. Then $\operatorname{Index}_{G, \mathbb{Z}} X \supseteq \operatorname{Index}_{G, \mathbb{Z}} Y$. Thus, the inclusion of indices of two $G$-spaces is a necessary condition for the existence of $G$-equivariant maps between these two spaces. If $E_{*}^{* * *}$ denotes the Serre spectral sequence of the Borel construction of $X$, then the homomorphism $\pi_{X}^{*}$ can be presented as the composition

$$
H^{*}(\mathrm{~B} G, \mathbb{Z}) \rightarrow E_{2}^{*, 0} \rightarrow E_{3}^{*, 0} \rightarrow E_{4}^{*, 0} \rightarrow \ldots \rightarrow E_{\infty}^{*, 0} \subseteq H^{*}\left(X \times_{G} \mathrm{E} G, \mathbb{Z}\right) .
$$

Since the $E_{2}$-term of the spectral sequence is given by $E_{2}^{p, q}=H^{p}\left(\mathrm{~B} G, H^{q}(X, \mathbb{Z})\right)$ the first step in the computation of the index is study of the cohomology $H^{*}(X, \mathbb{Z})$ as a $G$-module (Section [3.2). The final step is explicit description of non-zero differentials in the spectral sequence and application of the presentation (5) of the homomorphism $\pi_{X}^{*}$ (Section 3.3).

\subsection{The Index of $S\left(U_{4} \times U_{2}\right)$}

Let $V^{1}$ be the 1 -dimensional complex $\mathbb{Z}_{4}$-representation, or 2-dimensional real $\mathbb{Z}_{4}$-representation, induced by the correspondence $1 \mapsto e^{i \pi / 2}$. Then the 3 -dimensional real vector space $U_{4} \subset \mathbb{R}^{4}$ seen as a real $\mathbb{Z}_{4}$-representation decomposes into a sum of two irreducible real $\mathbb{Z}_{4}$-representations

$$
U_{4}=\operatorname{span}\{(1,0,-1,0),(0,1,0,-1)\} \oplus \operatorname{span}\{(1,-1,1,-1)\} \cong V^{1} \oplus U_{2} .
$$

Here "span" stands for all $\mathbb{R}$-linear combinations of the given vectors. It can be also seen that there is an isomorphism of real $\mathbb{Z}_{4}$-representations

$$
U_{4} \times U_{2} \cong V^{1} \oplus U_{2} \oplus U_{2} \cong V^{1} \oplus\left(V^{1} \otimes_{\mathbb{C}} V^{1}\right) .
$$

Here $V^{1} \otimes_{\mathbb{C}} V^{1}$ is a tensor product of complex representations and therefore a 1-dimensional complex $\mathbb{Z}_{4}$-representation or a 2 -dimensional real $\mathbb{Z}_{4}$-representation. Following [1, Section 8, p. 271 and Appendix, page 285] we deduce the total Chern class of the $\mathbb{Z}_{4}$-representation $U_{4} \times U_{2}$

$$
c\left(U_{4} \times U_{2}\right)=c\left(V^{1}\right) \cdot c\left(V^{1} \otimes V^{1}\right) .
$$

Therefore the top Chern class, or the Euler class of the underlying real representation, is

$$
c_{2}\left(U_{4} \times U_{2}\right)=c_{1}\left(V^{1}\right) \cdot c_{1}\left(V^{1} \otimes V^{1}\right)=c_{1}\left(V^{1}\right) \cdot\left(c_{1}\left(V^{1}\right)+c_{1}\left(V^{1}\right)\right)=2 U^{2} \in H^{*}\left(\mathbb{Z}_{4} ; \mathbb{Z}\right) .
$$

The $\mathbb{Z}_{4}$-index of the sphere $S\left(U_{4} \times U_{2}\right)$ is generated by the Euler class 2, Proposition $3.11]$, and so

$$
\operatorname{Index}_{\mathbb{Z}_{4}, \mathbb{Z}} S\left(U_{4} \times U_{2}\right)=\left\langle 2 U^{2}\right\rangle .
$$




\subsection{The cohomology $H^{*}(\Omega ; \mathbb{Z})$ as a $\mathbb{Z}_{4}$-module}

The cohomology is going to be determined via Poincaré-Lefschetz duality and an explicit study of cell structures for the spaces $X$ and $Y$.

Poincaré-Lefschetz duality [4, Theorem 70.2, page 415] implies that

$$
H^{*}(\Omega ; \mathbb{Z})=H^{*}(X \backslash Y ; \mathbb{Z}) \cong H_{8-*}(X, Y ; \mathbb{Z})
$$

and therefore we analyze the homology of the pair $(X, Y)$. The inclusion $Y \hookrightarrow X$ induces a map in homology. In particular, we consider this map in dimensions 2 and 4,

$$
\Phi: H_{2}(Y ; \mathbb{Z}) \rightarrow H_{2}(X ; \mathbb{Z}) \quad \text { and } \quad \Psi: H_{4}(Y ; \mathbb{Z}) \rightarrow H_{4}(X ; \mathbb{Z})
$$

The long exact sequence in homology of the pair $(X, Y)$ yields that the possibly non-zero homology groups of the pair $(X, Y)$ with $\mathbb{Z}$-coefficients are

$$
H_{i}(X, Y ; \mathbb{Z})= \begin{cases}\mathbb{Z}\left[\mathbb{Z}_{4}\right] / \operatorname{im} \Phi, & i=2 \\ \operatorname{ker} \Phi, & i=3 \\ \mathbb{Z}\left[\mathbb{Z}_{4}\right] \oplus \mathbb{Z}\left[\mathbb{Z}_{4} / \mathbb{Z}_{2}\right] / \operatorname{im} \Psi, & i=4 \\ \operatorname{ker} \Psi, & i=5 \\ \mathbb{Z}\left[\mathbb{Z}_{4}\right], & i=6 \\ \mathbb{Z}, & i=8\end{cases}
$$

Thus explicit formulas for the maps $\Phi$ and $\Psi$ are needed in order to determine the homology $H_{*}(X, Y ; \mathbb{Z})$ and its exact $\mathbb{Z}_{4}$-module structure.

Let $x_{1}, x_{2}, x_{3}, x_{4} \in H_{2}(X ; \mathbb{Z})$ be generators carried by individual copies of $S^{2}$ in the product $X=S^{2} \times S^{2} \times S^{2} \times S^{2}$. The generator of the group $\mathbb{Z}_{4}=\langle\omega\rangle$ acts on this basis of $H_{2}(X ; \mathbb{Z})$ by $\omega \cdot x_{i}=x_{i+1}$ where $x_{5}=x_{1}$. Then by $x_{i} x_{j} \in H_{4}(X ; \mathbb{Z}), i \neq j$, we denote the generator carried by the product of $i$-th and $j$-th copy of $S^{2}$ in $X$. The action of $\omega$ on $H_{4}(X ; \mathbb{Z})$ is described by

$$
x_{1} x_{2} \stackrel{\cdot \omega}{\longmapsto} x_{2} x_{3} \stackrel{\cdot \omega}{\longmapsto} x_{3} x_{4} \stackrel{\cdot \omega}{\longmapsto} x_{1} x_{4} \quad \text { and } \quad x_{1} x_{3} \stackrel{\cdot \omega}{\longmapsto} x_{2} x_{4} .
$$

Let similarly $y_{1}, y_{2} \in H_{2}(X ; \mathbb{Z})$ be generators carried by individual copies of $S^{2}$ in the product $Y=S^{2} \times S^{2}$. Then $\omega \cdot y_{1}=y_{2}$ and $\omega \cdot y_{2}=y_{1}$. Again $y_{1} y_{2}$ denotes the generator of $H_{4}(Y ; \mathbb{Z})$ and $\omega \cdot y_{1} y_{2}=y_{1} y_{2}$. Note that $\omega$ preserves the orientations of $X$ and $Y$ and therefore acts trivially on $H_{8}(X ; \mathbb{Z})$ and on $H_{4}(Y ; \mathbb{Z})$.

The inclusion $Y \subset X$ induces a map in homology $H_{*}(X ; \mathbb{Z}) \subset H_{*}(Y ; \mathbb{Z})$, which in dimensions 2 and 4 is given by

$$
\begin{aligned}
& y_{1} \longmapsto x_{1}+x_{3}, \quad y_{2} \longmapsto x_{2}+x_{4}, \\
& y_{1} y_{2} \longmapsto x_{1} x_{2}+x_{2} x_{3}+x_{3} x_{4}+x_{1} x_{4} .
\end{aligned}
$$

This can be seen from the dual cohomology picture: An element is mapped to a sum of generators intersecting its image, with appropriately attached intersection numbers. 
Thus $\Phi$ and $\Psi$ are injective and

$$
\operatorname{im} \Phi=\left\langle x_{1}+x_{3}, x_{2}+x_{4}\right\rangle, \quad \operatorname{im} \Psi=\left\langle x_{1} x_{2}+x_{2} x_{3}+x_{3} x_{4}+x_{1} x_{4}\right\rangle .
$$

Let $N=\mathbb{Z} \oplus \mathbb{Z}$ be the $\mathbb{Z}_{4}$-representation given by $\omega \cdot(a, b)=(b,-a)$, while $M$ denotes the representation $\mathbb{Z}\left[\mathbb{Z}_{4}\right] /\left(1+\omega+\omega^{2}+\omega^{3}\right) \mathbb{Z}$. Then the non-trivial cohomology of the space $X \backslash Y$, as a $\mathbb{Z}_{4}$-module via the isomorphism (7), is given by

$$
H^{i}(\Omega ; \mathbb{Z})= \begin{cases}N, & i=6 \\ M \oplus \mathbb{Z}\left[\mathbb{Z}_{4} / \mathbb{Z}_{2}\right], & i=4 \\ \mathbb{Z}\left[\mathbb{Z}_{4}\right], & i=2 \\ \mathbb{Z}, & i=0\end{cases}
$$

\subsection{The Serre spectral sequence of $\Omega \times_{\mathbb{Z}_{4}} \mathrm{EZ}_{4}$}

The Serre spectral sequence associated to the fibration $\Omega \rightarrow \Omega \times_{\mathbb{Z}_{4}} \mathrm{EZ}_{4} \rightarrow \mathrm{B} \mathbb{Z}_{4}$ is a spectral sequence with non-trivial local coefficients, since $\pi_{1}\left(\mathrm{~B} \mathbb{Z}_{4}\right)=\mathbb{Z}_{4}$ acts non-trivially (8) on the cohomology $H^{*}(\Omega ; \mathbb{Z})$. The first step in the study of such a spectral sequence is to understand the $H^{*}\left(\mathbb{Z}_{4} ; \mathbb{Z}\right)$-module structure on the rows of its $E_{2}$-term.

The $E_{2}$-term of the sequence is given by

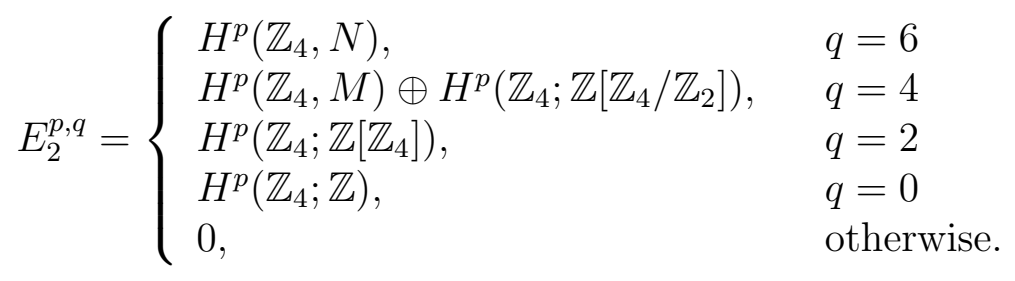

Lemma 3.1. $H^{p}\left(\mathbb{Z}_{4} ; \mathbb{Z}\left[\mathbb{Z}_{4}\right]\right)= \begin{cases}\mathbb{Z}, & p=0 \\ 0, & p>0\end{cases}$

and multiplication by $U \in H^{2}\left(\mathbb{Z}_{4} ; \mathbb{Z}\right)$ is trivial, $U \cdot H^{p}\left(\mathbb{Z}_{4} ; \mathbb{Z}\left[\mathbb{Z}_{4}\right]\right)=0$.

For the proof one can consult [3, Example 2, page 58].

Lemma 3.2. $H^{*}\left(\mathbb{Z}_{4} ; \mathbb{Z}\left[\mathbb{Z}_{4} / \mathbb{Z}_{2}\right]\right) \cong H^{*}\left(\mathbb{Z}_{2} ; \mathbb{Z}\right)$, where the module structure is given by the restriction homomorphism $\operatorname{res}_{\mathbb{Z}_{2}}^{\mathbb{Z}_{4}}: H^{*}\left(\mathbb{Z}_{4} ; \mathbb{Z}\right) \rightarrow H^{*}\left(\mathbb{Z}_{2} ; \mathbb{Z}\right)$.

In other words, if we denote $H^{*}\left(\mathbb{Z}_{2} ; \mathbb{Z}\right)=\mathbb{Z}[T] / 2 T$, $\operatorname{deg} T=2$, then $\operatorname{res}_{\mathbb{Z}_{2}}^{\mathbb{Z}_{4}}(U)=T$ and consequently:

(A) $H^{*}\left(\mathbb{Z}_{4} ; \mathbb{Z}\left[\mathbb{Z}_{4} / \mathbb{Z}_{2}\right]\right)$ is generated by one element of degree 0 as a $H^{*}\left(\mathbb{Z}_{4} ; \mathbb{Z}\right)$-module, and

(B) multiplication by $U$ in $H^{*}\left(\mathbb{Z}_{4} ; \mathbb{Z}\left[\mathbb{Z}_{4} / \mathbb{Z}_{2}\right]\right)$ is an isomorphism, while multiplication by $2 U$ is zero.

The proof is a direct application of Shapiro's lemma [3. (6.3), page 73] and a small part of the restriction diagram [2, Section 4.5.2].

Lemma 3.3. There exists an element $\Lambda \in H^{*}\left(\mathbb{Z}_{4}, M\right)$ of degree 1 such that $4 \Lambda=0$ and $H^{*}\left(\mathbb{Z}_{4}, M\right) \cong H^{*}\left(\mathbb{Z}_{4} ; \mathbb{Z}\right) \cdot \Lambda$ as an $H^{*}\left(\mathbb{Z}_{4} ; \mathbb{Z}\right)$-module. 
Proof. The short exact sequence of $\mathbb{Z}_{4}$-modules

$$
0 \longrightarrow \mathbb{Z}^{1+\omega+\omega^{2}+\omega^{3}} \mathbb{Z}\left[\mathbb{Z}_{4}\right] \longrightarrow M \longrightarrow 0
$$

induces a long exact sequence in cohomology [3. Proposition 6.1, page 71], which is natural with respect to $H^{*}\left(\mathbb{Z}_{4} ; \mathbb{Z}\right)$-module multiplication. Since $\mathbb{Z}\left[\mathbb{Z}_{4}\right]$ is a free module we get enough zeros to recover the information we need:

$$
\begin{aligned}
& \begin{array}{c}
0 \longrightarrow H^{0}\left(\mathbb{Z}_{4} ; \mathbb{Z}\right) \stackrel{\xi}{\mathbb{Z}} H^{0}\left(\mathbb{Z}_{4} ; \mathbb{Z}\left[\mathbb{Z}_{4}\right]\right) \\
\mathbb{Z}
\end{array} \longrightarrow H^{0}\left(\mathbb{Z}_{4}, M\right) \longrightarrow H^{1}\left(\mathbb{Z}_{4} ; \mathbb{Z}\right) \longrightarrow \\
& \longrightarrow \begin{array}{cc}
H^{1}\left(\mathbb{Z}_{4} ; \mathbb{Z}\left[\mathbb{Z}_{4}\right]\right) \\
0
\end{array} H^{1}\left(\mathbb{Z}_{4}, M\right) \longrightarrow H^{2}\left(\mathbb{Z}_{4} ; \mathbb{Z}\right) \longrightarrow
\end{aligned}
$$

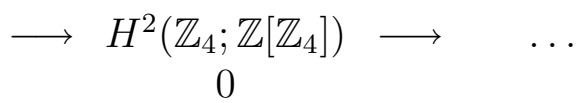

$$
\begin{aligned}
& \longrightarrow H^{i}\left(\mathbb{Z}_{4} ; \mathbb{Z}\left[\mathbb{Z}_{4}\right]\right) \longrightarrow H^{i}\left(\mathbb{Z}_{4}, M\right) \longrightarrow H^{i+1}\left(\mathbb{Z}_{4} ; \mathbb{Z}\right) \longrightarrow \\
& 0 \\
& \longrightarrow H^{i+1}\left(\mathbb{Z}_{4} ; \mathbb{Z}\left[\mathbb{Z}_{4}\right]\right) \longrightarrow \quad \cdots \\
& 0
\end{aligned}
$$

The map $\xi: H^{0}\left(\mathbb{Z}_{4} ; \mathbb{Z}\right) \cong \mathbb{Z}^{\mathbb{Z}_{4}} \rightarrow H^{0}\left(\mathbb{Z}_{4} ; \mathbb{Z}\left[\mathbb{Z}_{4}\right]\right) \cong \mathbb{Z}\left[\mathbb{Z}_{4}\right]^{\mathbb{Z}_{4}}$ is a surjection. Indeed, $\xi$ is induced by the map $\mathbb{Z}_{1-1 \text { and onto }}^{\stackrel{1+\omega+\omega^{2}+\omega^{3}}{\longrightarrow}} \mathbb{Z}\left[\mathbb{Z}_{4}\right]^{\mathbb{Z}_{4}} \hookrightarrow \mathbb{Z}\left[\mathbb{Z}_{4}\right]$ which bijectively factorizes through the invariants of $\mathbb{Z}\left[\mathbb{Z}_{4}\right]$.

Lemma 3.4. There exists an element $\Upsilon \in H^{*}\left(\mathbb{Z}_{4}, N\right)$ of degree 1 such that $2 \Upsilon=0$ and $H^{*}\left(\mathbb{Z}_{4}, N\right) \cong H^{*}\left(\mathbb{Z}_{4} ; \mathbb{Z}\left[\mathbb{Z}_{4} / \mathbb{Z}_{2}\right]\right) \cdot \Upsilon$ as an $H^{*}\left(\mathbb{Z}_{4} ; \mathbb{Z}\right)$-module.

Proof. There is a short exact sequence of $\mathbb{Z}_{4}$-modules

$$
0 \rightarrow N \stackrel{\alpha}{\rightarrow} \mathbb{Z}\left[\mathbb{Z}_{4}\right] \stackrel{\beta}{\rightarrow} L \rightarrow 0
$$

where $L=\mathbb{Z}\left[\mathbb{Z}_{4}\right] / N$ and $\alpha(p, q)=(p, q,-p,-q)$. The map $\alpha$ is well defined because the following diagram commutes

$$
\begin{array}{ccccc}
N={ }_{a b} \mathbb{Z} \oplus \mathbb{Z} \ni & (p, q) & \stackrel{\alpha}{\longrightarrow} & (p, q,-p,-q) & \in \mathbb{Z}\left[\mathbb{Z}_{4}\right] \\
& \downarrow \cdot \omega & & \downarrow \cdot \omega & \\
N={ }_{a b} \mathbb{Z} \oplus \mathbb{Z} \ni & (q,-p) & \stackrel{\alpha}{\longrightarrow} & (q,-p,-q, p) & \in \mathbb{Z}\left[\mathbb{Z}_{4}\right] .
\end{array}
$$

The $\mathbb{Z}_{4}$-module $L$ is isomorphism to $\mathbb{Z}\left[\mathbb{Z}_{4} / \mathbb{Z}_{2}\right] \cong \mathbb{Z} \oplus \mathbb{Z}$ and the map $\beta$ is given, on generators, by

$$
(1,0,0,0) \longmapsto(1,0),(0,1,0,0) \longmapsto(0,1),(0,0,1,0) \longmapsto(1,0),(0,0,0,1) \longmapsto(1,0) .
$$

Therefore, the induced map of invariants $\mathbb{Z} \cong \mathbb{Z}\left[\mathbb{Z}_{4}\right]^{\mathbb{Z}_{4}} \stackrel{\beta}{\rightarrow} \mathbb{Z}\left[\mathbb{Z}_{4} / \mathbb{Z}_{2}\right] \cong \mathbb{Z}$ is a multiplication by 2 . Now, the long exact sequence in group cohomology [3, Prop. 6.1, p 71] implies the result. 


\begin{tabular}{|c|c|c|c|c|c|c|c|c|}
\hline 6 & 0 & $\Upsilon^{\Upsilon_{\mathbb{Z}_{2}}}$ & 0 & $T \Upsilon_{\mathbb{Z}_{2}}$ & 0 & $T^{2} \Upsilon_{\mathbb{Z}_{2}}$ & 0 & $T^{3} \Upsilon_{\mathbb{Z}_{2}}$ \\
\hline 5 & 0 & 0 & 0 & 0 & 0 & 0 & 0 & 0 \\
\hline 4 & \begin{tabular}{l|l}
$\mathbb{Z}_{2}$ & 0 \\
\end{tabular} & \begin{tabular}{|l|l}
0 & $\Lambda$ \\
$\mathbb{Z}$ \\
\end{tabular} & \begin{tabular}{l|l}
$T$ & 0 \\
$\mathbb{Z}_{2}$ & 0 \\
\end{tabular} & \begin{tabular}{|l|l|}
0 & $\mathbb{Z}_{4}$ \\
\end{tabular} & \begin{tabular}{|l|l}
$T^{2}$ & 0 \\
$\mathbb{Z}_{2}$ & 0 \\
\end{tabular} & \begin{tabular}{|l|l|l|} 
& $\Lambda U^{2}$ \\
$\mathbb{Z}_{4}$
\end{tabular} & \begin{tabular}{l|l}
$T^{3}$ & 0 \\
$\mathbb{Z}_{2}$ & 0 \\
$y_{2}$
\end{tabular} & \begin{tabular}{|l|l|l|l}
$\Lambda U^{3}$ \\
$\mathbb{Z}_{4}$ \\
\end{tabular} \\
\hline 3 & 0 & 0 & 0 & 0 & 0 & 0 & 0 & 0 \\
\hline 2 & $\mathbb{Z}$ & 0 & 0 & 0 & 0 & 0 & 0 & 0 \\
\hline 1 & 0 & 0 & 0 & 0 & 0 & 0 & 0 & 0 \\
\hline 0 & $1_{\mathbb{Z}}$ & 0 & $U_{\mathbb{Z}_{4}}$ & 0 & $U_{\mathbb{Z}_{4}}^{2}$ & 0 & $U_{\mathbb{Z}_{4}}^{3}$ & 0 \\
\hline
\end{tabular}

Figure 2: The $E_{2}$-term

The $E_{2}$-term of the Borel construction $(X \backslash Y) \times_{\mathbb{Z}_{4}} \mathrm{E}_{4}$, with the $H^{*}\left(\mathbb{Z}_{4} ; \mathbb{Z}\right)$-module structure, is presented in Figure 2

The differentials of the spectral sequence are retrieved from the fact that the $\mathbb{Z}_{4}$ action on $\Omega$ is free. Therefore $H_{\mathbb{Z}_{4}}^{i}(\Omega ; \mathbb{Z})=0$ for all $i>8$. Since the spectral sequence is converging to the graded group associated with $H_{\mathbb{Z}_{4}}^{i}(\Omega ; \mathbb{Z})$ this means that for $p+q>8$ nothing survives. Thus the only non-zero second differentials are $d_{2}: E_{2}^{2 i+1,6} \rightarrow E_{2}^{2 i+4,4}$, $d_{2}\left(T^{i} \Upsilon\right)=T^{i+1}, i>0$, as displayed in Figure 3 ,

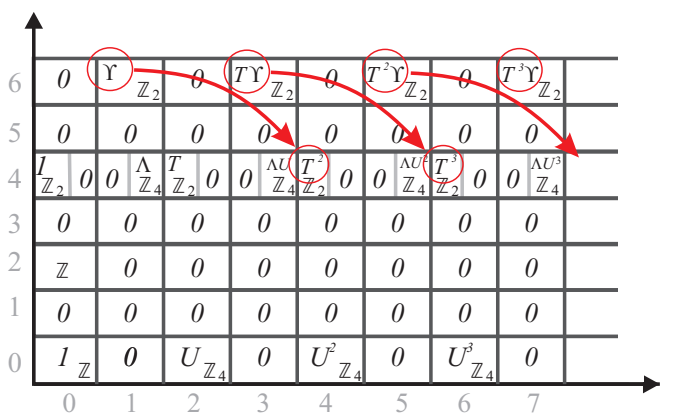

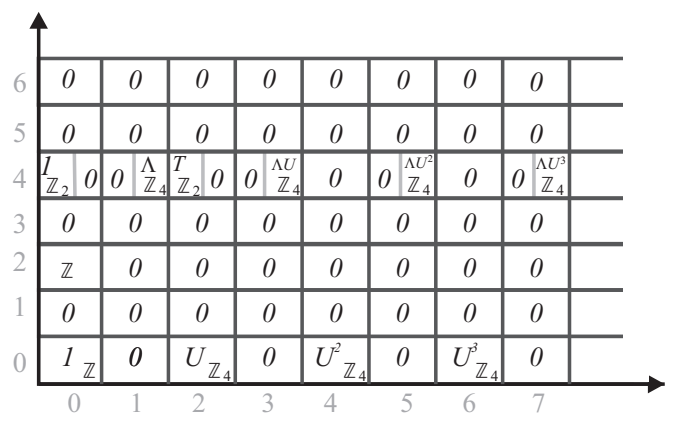

Figure 3: Differentials in $E_{2}$ and $E_{3}$-terms

The last remaining non-zero differentials are $d_{4}: E_{4}^{2 i+1,4} \rightarrow E_{4}^{2 i+6,0}, d_{6}\left(U^{i} \Lambda\right)=U^{i+3}$, $i>0$. Then $E_{5}=E_{\infty}, c f$. Figure 4 .

\subsection{The index of $\Omega$}

The conclusion $d_{6}(\Lambda)=U^{3}$ implies that

$$
\text { Index }_{\mathbb{Z}_{4}, \mathbb{Z}} \Omega=\left\langle U^{3}\right\rangle .
$$

Since the generator $2 U^{2}$ of the Index $\mathbb{Z}_{4}, \mathbb{Z} S\left(U_{4} \times U_{2}\right)$ is not contained in Index $\mathbb{Z}_{4}, \mathbb{Z} \Omega$ it follows that there is no $\mathbb{Z}_{4}$-equivariant map $\Omega \rightarrow S\left(U_{4} \times U_{2}\right)$. This concludes the proof of Theorem 2.2. 

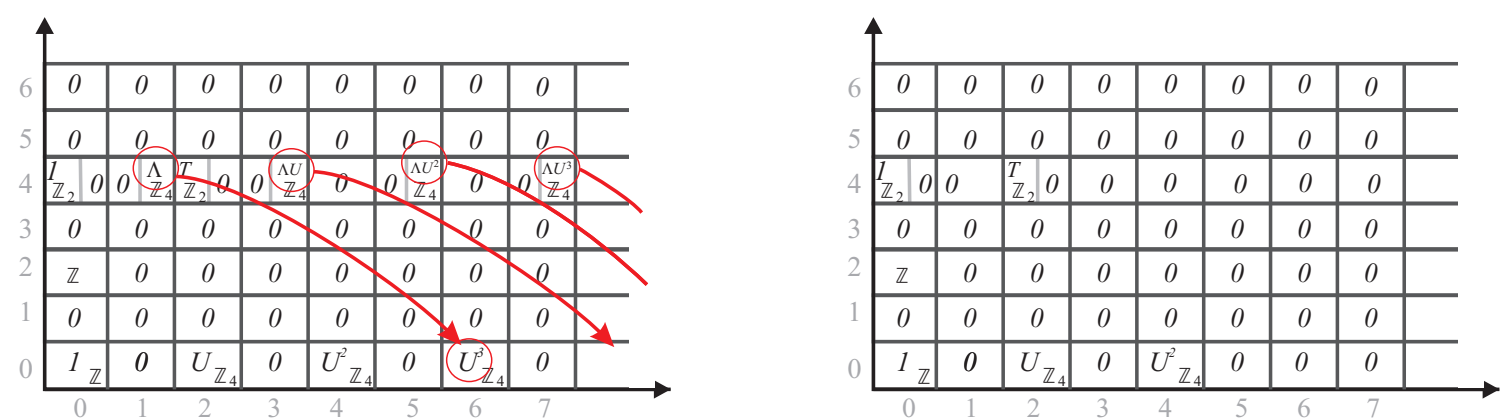

Figure 4: Differentials in $E_{4}$ and $E_{5}$-terms

Remark 3.5. As one of the referees observed, in order to prove Theorem 2.2 there was no need to compute the Index $\mathbb{Z}_{\mathbb{Z}_{4}, \mathbb{Z}} \Omega$. The structure of $E_{2}$-term (9) of the spectral sequence of the fibration $\Omega \times_{\mathbb{Z}_{4}} \mathrm{EZ}_{4}$ and Lemma 3.1 guarantee that the elements $U^{2}$ and $2 U^{2}$ survive to $E_{\infty}$-term. This provides the contradiction since $\operatorname{Index}_{\mathbb{Z}_{4}, \mathbb{Z}} S\left(U_{4} \times U_{2}\right)=\left\langle 2 U^{2}\right\rangle$.

\section{Concluding remarks}

\subsection{The $\mathbb{F}_{2}$-index}

Let $H^{*}\left(\mathbb{Z}_{4}, \mathbb{F}_{2}\right)=\mathbb{F}_{2}[e, u] / e^{2}, \operatorname{deg}(e)=1, \operatorname{deg}(u)=2$. The homomorphism of coefficients $j: \mathbb{Z} \rightarrow \mathbb{F}_{2}, j(1)=1$, induces a homomorphism in group cohomology $j^{*}: H^{*}\left(\mathbb{Z}_{4} ; \mathbb{Z}\right) \rightarrow$ $H^{*}\left(\mathbb{Z}_{4}, \mathbb{F}_{2}\right)$ given by $j^{*}(U)=u$ (compare [2, Section 4.5.2]).

The $\mathbb{F}_{2}$-index of the configuration space $\Omega$ is

$$
\operatorname{Index}_{\mathbb{Z}_{4}, \mathbb{F}_{2}} \Omega=\left\langle e u^{2}, u^{3}\right\rangle .
$$

This can be obtained in a similar fashion as we obtained the index with $\mathbb{Z}$-coefficients in Section 3.3. The relevant $E_{2}$-term of the Serre spectral sequence of the fibration $\Omega \rightarrow \Omega \times_{\mathbb{Z}_{4}} \mathrm{EZ}_{4} \rightarrow \mathrm{BZ}_{4}$ is described in Figure 5 .

The $\mathbb{F}_{2}$-index of the sphere $S\left(U_{4} \times U_{2}\right)$ is generated by the $j^{*}$ image of the generator

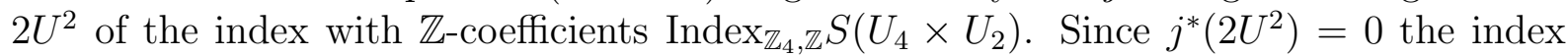
Index $_{\mathbb{Z}_{4}, \mathbb{F}_{2}} S\left(U_{4} \times U_{2}\right)$ is trivial. Therefore, for our problem no conclusion can be obtained from the study of the $\mathbb{F}_{2}$-index. The same observation holds even when the complete group $D_{8}$ is used. The $\mathbb{F}_{2}$-index of the sphere $S\left(U_{4} \times U_{2}\right)$ would be generated by $x y w=0 \in H^{*}\left(D_{8} ; \mathbb{F}_{2}\right)$, in the notation of [2].

\subsection{The square peg problem}

The method of configuration spaces can also be set up for to the continuous square peg problem. Following the ideas presented in Section 2 taking for $X$ the product $S^{1} \times S^{1} \times$ $S^{1} \times S^{1}$, for $Y$ the subspace $Y=\left\{(x, y, x, y) \mid x, y \in S^{1}\right\}$ and for the configuration space 


\begin{tabular}{|c|c|c|c|c|c|c|c|c|}
\hline 6 & 1 & $t$ & $t^{2}$ & $t^{3}$ & $t^{4}$ & $t^{5}$ & $t^{6}$ & $t^{7}$ \\
\hline 5 & 0 & 0 & 0 & 0 & 0 & 0 & 0 & 0 \\
\hline 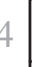 & $1 e$ & \begin{tabular}{|l|l}
$t$ & $u$ \\
\end{tabular} & $\begin{array}{lll}t^{2} & e u\end{array}$ & \begin{tabular}{|l|l|}
$t^{3}$ & $u^{2}$ \\
\end{tabular} & $t^{4} e u^{2}$ & $t^{5} u^{3}$ & $t^{6} e u^{3}$ & \begin{tabular}{|l|l|}
$t^{7}$ & $u^{4}$ \\
\end{tabular} \\
\hline & 0 & 0 & 0 & 0 & 0 & 0 & 0 & 0 \\
\hline & $F_{2}$ & 0 & 0 & 0 & 0 & 0 & 0 & 0 \\
\hline 1 & 0 & 0 & 0 & 0 & 0 & 0 & 0 & 0 \\
\hline 0 & 1 & $e$ & $u$ & $e u$ & $u^{2}$ & $e u^{2}$ & $u^{3}$ & $e u^{3}$ \\
\hline
\end{tabular}

Figure 5: $E_{2}$-term with $\mathbb{F}_{2}$-coefficients

$\Omega=X \backslash Y$, the square peg problem can be related to the question of the existence of a $D_{8}$-equivariant map $\Omega \rightarrow S\left(U_{4} \times U_{2}\right)$. The Fadell-Husseini indexes can be retrieved:

$$
\operatorname{Index}_{\mathbb{Z}_{4}, \mathbb{Z}} \Omega=\left\langle U^{2}\right\rangle \quad \text { and } \quad \operatorname{Index~}_{\mathbb{Z}_{4}, \mathbb{Z}} S\left(U_{4} \times U_{2}\right)=\left\langle 2 U^{2}\right\rangle,
$$

but since Index $\mathbb{Z}_{4}, \mathbb{Z} \Omega \supseteq \operatorname{Index}_{\mathbb{Z}_{4}, \mathbb{Z}} S\left(U_{4} \times U_{2}\right)$ the result does not yield any conclusion. The same can be done for the complete symmetry group $D_{8}$, explicitly Index $D_{8}, \mathbb{Z} S\left(U_{4} \times U_{2}\right)=$ $\langle 2 \mathcal{W}\rangle$ and $\mathcal{W} \in \operatorname{Index}_{D_{8}, \mathbb{Z}} \Omega$.

\section{Acknowledgements.}

Thanks to Anton Dochterman and to the referees for many interesting and useful comments.

\section{References}

[1] M. F. AtiYah, Characters and cohomology of finite groups, Inst. Hautes Études Sci. Publ. Math. No. 9 (1961), 23-64.

[2] P. V. M. Blagojević, G. M. Ziegler, The ideal-valued index for a dihedral group action, and mass partition by two hyperplanes, preprint, revised version arXiv:0704.1943v2, July 2008, 42 pages.

[3] K. S. Brown, Cohomology of Groups, Graduate Texts in Math. 87, Springer-Verlag, New York, Berlin, 1982.

[4] J. R. Munkres, Elements of Algebraic Topology, Addison-Wesley, Menlo Park CA, 1984.

[5] L. G. Shnirelman, On certain geometrical properties of closed curves (in Russian), Uspehi Matem. Nauk 10 (1944), 34-44, http://tinyurl.com/28gsy3.

[6] Y. Soibelman, Topological Borsuk problem, Preprint 2002, 4 pages, http://arXiv.org/abs/math/0208221v2. 